The Astrophysical Journal, 540:L53-L56, 2000 September 1

(C) 2000. The American Astronomical Society. All rights reserved. Printed in U.S.A.

\title{
STAR FORMATION IN CLUSTERS: EARLY SUBCLUSTERING IN THE SERPENS CORE
}

\author{
Leonardo Testi, ${ }^{1,2}$ Anneila I. Sargent, ${ }^{2}$ Luca Olmi, ${ }^{3}$ and Joseph S. Onello ${ }^{4}$ \\ Received 2000 March 3; accepted 2000 May 24; published 2000 August 22
}

\begin{abstract}
We present high-resolution interferometric and single-dish observations of molecular gas in the Serpens clusterforming core. Star formation does not appear to be homogeneous throughout the core, but is localized in spatially and kinematically separated subclusters. The stellar (or protostellar) density in each of the subclusters is much higher than the mean for the entire Serpens cluster. This is the first observational evidence for the hierarchical fragmentation of protocluster cores suggested by cluster formation models.
\end{abstract}

Subject headings: ISM: clouds — radio continuum: ISM — stars: formation

\section{INTRODUCTION}

It is generally accepted that most stars are born in clusters (see Clarke, Bonnell, \& Hillenbrand 2000). The way in which clusters form and evolve is therefore likely to influence the distribution of masses for stars in the field, the initial mass function (IMF; Salpeter 1955; Scalo 1986). In very young, embedded clusters the distribution of stellar masses is often similar to the IMF (Palla \& Stahler 1999; Meyer et al. 2000), and the mass spectra of prestellar and protostellar condensations in the Serpens and $\rho$ Ophiuchi cluster-forming cores are also consistent with the IMF (Testi \& Sargent 1998, hereafter TS98; Motte, André, \& Neri 1998).

It has been suggested that within stellar clusters star formation occurs preferentially in subclusters in which the stellar density is much enhanced (Clarke et al. 2000). This has important implications for cluster evolution. For example, more massive stars could be produced by coalescence (Stahler, Palla, $\&$ Ho 2000). To date, there is little evidence for subclustering in the Orion Nebula cluster (Bate, Clarke, \& McCaughrean 1998 ) or in the smaller clusters around intermediate-mass pre-main-sequence stars (Testi, Palla, \& Natta 1999).

However, models advocate subclusters with a much higher stellar density at the time of formation (Bonnell, Bate, \& Zinnecker 1998). It is therefore important to establish if subclustering is present in the very youngest clusters. The mean stellar densities and the stellar-to-gas mass ratio in such subclusters can provide critical observational constraints on coalescence, competitive accretion, and binary evolution models (Bonnell et al. 1997, 1998; Kroupa, Petr, \& McCaughrean 1999).

The Serpens molecular cloud, at $\sim 310$ pc (de Lara et al. 1991 ), is one of the most active nearby cluster-forming cores. Inside the 500-1500 $M_{\odot}, \sim 0.6 \mathrm{pc}$ diameter cloud of molecular gas (White, Casali, \& Eiroa 1995) is a young protocluster comprising about 100 embedded young stellar objects (YSOs), protostars, and prestellar clumps (Strom, Vrba, \& Strom 1976; Eiroa \& Casali 1992; Giovannetti et al. 1998; Kaas 1999; Casali, Eiroa, \& Duncan 1993; Hurt \& Barsony 1996; TS98). Numerous jets and molecular outflows have also been detected (Rodríguez et al. 1989; White et al. 1995; Eiroa et al. 1997;

\footnotetext{
${ }^{1}$ Osservatorio Astrofisico di Arcetri, Largo E. Fermi 5, I-50125 Firenze, Italy; 1testi@arcetri.astro.it.

${ }^{2}$ Division of Physics, Mathematics, and Astronomy, California Institute of Technology, MS 105-24, Pasadena, CA 91125.

${ }^{3}$ Department of Physics, State University of New York, Cortland, NY 13045.

${ }^{4}$ Large Millimeter Telescope Project and Five College Radio Astronomy Observatory, University of Massachusetts, 630 Lederle Graduate Research Center, Amherst, MA 01003.
}

Herbst, Beckwith, \& Robberto 1997; Wolf-Chase et al. 1998; Davis et al. 1999; Hodapp 1999; Hogerheijde et al. 1999). The total estimated mass of the YSOs, protostars, and prestellar clumps is in the range 40-80 $M_{\odot}$ (Giovannetti et al. 1998; TS98), implying an overall star formation efficiency of $2 \%-5 \%$, similar to most nearby molecular clouds. The protocluster radius $(\sim 0.2 \mathrm{pc})$ and mean stellar density $\left(\sim 400-800\right.$ stars pc $\left.^{-3}\right)$ are typical of very young embedded clusters (Testi et al. 1999), making this an ideal laboratory for studying early cluster formation processes. Here we present wide-field, high-resolution, aperture synthesis and single-dish millimeter-wave molecular line observations of the Serpens core that support the concept of at-birth subclustering.

\section{OBSERVATIONS AND RESULTS}

Owens Valley Radio Observatory (OVRO) millimeter-wave array mosaic observations of the 5.5 $\times 5.5$ inner region of the Serpens molecular core in the CS (2-1) transition at $97.98 \mathrm{GHz}$ were obtained at the same time as the $3 \mathrm{~mm}$ continuum data described by TS 98 . Details of the observations, data reduction, and mosaic construction are described elsewhere (TS98; Testi \& Sargent 2000). Spectral resolution was $0.4 \mathrm{~km} \mathrm{~s}^{-1}$ over a $24 \mathrm{~km} \mathrm{~s}^{-1}$ bandwidth centered at $V_{\mathrm{LSR}}=8 \mathrm{~km} \mathrm{~s}^{-1}$. The synthesized beam is 5".5 $\times 4.3$ (FWHM), and the noise level in each channel of the final cleaned mosaic cube is $\sim 140 \mathrm{mJy}$ beam $^{-1}$. During spring/fall 1998, additional observations of SMM4, S68N, and the CS6, CS7, and CS8 features identified in Figure $1 a$ were acquired (Testi \& Sargent 2000). The digital correlator was configured so that the $\mathrm{N}_{2} \mathrm{H}^{+}(1-0)$ transition at 93.2 $\mathrm{GHz}$ and the $\mathrm{CH}_{3} \mathrm{OH}\left(2_{-1}-1_{-1}\right) \mathrm{E} 2$ and $\left(2_{0}-1_{0}\right) \mathrm{A}^{+}$transitions at $96.7 \mathrm{GHz}$ were detected simultaneously in the lower and upper sidebands, respectively. Spectral resolution was 0.4 $\mathrm{km} \mathrm{s}^{-1}$ over a $25 \mathrm{~km} \mathrm{~s}^{-1}$ band. Calibration was carried out as in TS98. The data sets were imaged using the AIPS IMAGR task.

A $10^{\prime} \times 10^{\prime}$ region of the Serpens cloud core, centered on $\alpha=18^{\mathrm{h}} 27^{\mathrm{m}} 20^{\mathrm{s}}, \delta=1^{\circ} 12^{\prime} 30^{\prime \prime}$ (B1950.0), was also mapped in the $\mathrm{N}_{2} \mathrm{H}^{+}(1-0)$ line in 1999 March and October using the $13.7 \mathrm{~m}$ telescope of the Five College Radio Astronomy Observatory ${ }^{6}$ (FCRAO) and the SEQUOIA focal plane array. For the one-beam sampled maps, the half-power beam width was $\sim 52^{\prime \prime}$. Low-noise InP MMIC-based amplifiers resulted in mean receiver temperature of $70 \mathrm{~K}$ (single sideband) and system

\footnotetext{
${ }^{6}$ The Five College Radio Astronomy Observatory is operated with support from the National Science Foundation and with permission of the Metropolitan District Commission.
} 

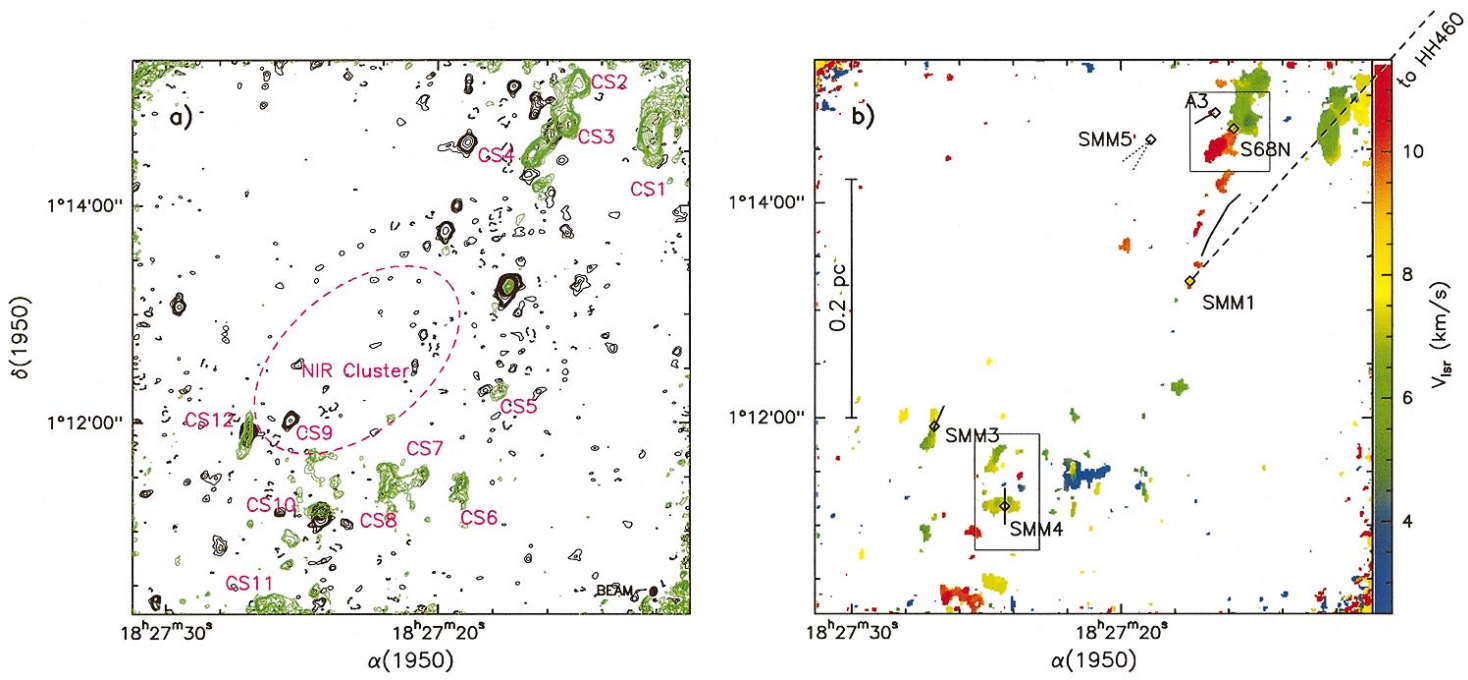

FIG. 1.-(a) Integrated intensity contours for CS (2-1) emission in the Serpens core are shown in green, overlaid on the 3 mm continuum map (TS98). Contour levels begin at $0.9 \mathrm{Jy} \mathrm{km} \mathrm{s}^{-1}$ beam ${ }^{-1}(3 \sigma)$ and are spaced by 0.3 to 3 and thereafter by $1 \mathrm{Jy} \mathrm{km} \mathrm{s}^{-1}$ beam $^{-1}$. The dashed ellipse represents the near-IR cluster (Kaas 1999). The CS features discussed in the text are marked CS1 to CS12. (b) Color-coded map of the mean velocity variations across the CS structures of (a). Known outflow sources, represented by open diamonds, are labeled, and solid lines indicate the orientations of observed jets or, for SMM5, reflection nebulosity. The dashed line from SMM1 indicates the direction to HH 460. The two rectangles mark the areas shown in Fig. 2. Increased noise in the upper left and lower right corners of both images is due to loss of sensitivity at the edge of the mosaic.
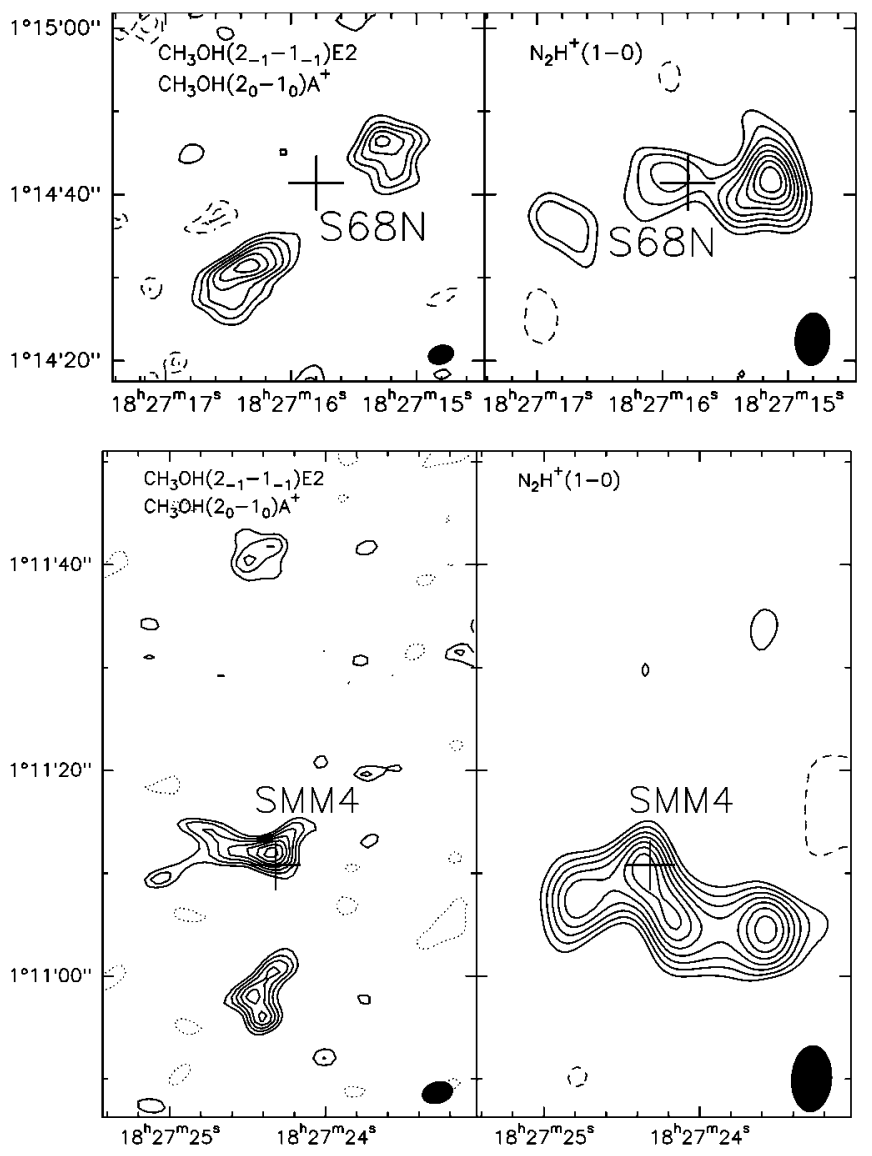

FIG. 2.-Integrated intensity maps of $\mathrm{CH}_{3} \mathrm{OH}\left(2_{k}-1_{k}\right)$ and $\mathrm{N}_{2} \mathrm{H}^{+}(1-0)$ for S68N and SMM4 the two regions indicated in Fig. 1b. Synthesized beams (FWHM) are shown by black ellipses in the lower right corners. Contour levels start at $3 \sigma$ and are spaced by $1 \sigma\left(0.3 \mathrm{Jy} \mathrm{km} \mathrm{s}^{-1}\right.$ beam $^{-1}$ for $\mathrm{S} 68 \mathrm{~N}$ and 0.2 Jy $\mathrm{km} \mathrm{s}^{-1}$ beam ${ }^{-1}$ for SMM4). temperatures of $160-250 \mathrm{~K}$. The spectrometer was an autocorrelator with $24 \mathrm{kHz}\left(0.077 \mathrm{~km} \mathrm{~s}^{-1}\right)$ spectral resolution and $20 \mathrm{MHz}$ bandwidth. Typical integration times were 5-15 minutes in frequency-switching mode, with a throw of $8 \mathrm{MHz}$. The main-beam efficiency, to convert the antenna temperature to brightness temperature $\eta_{\mathrm{mb}}$, is 0.51 , and the final $\mathrm{rms}$ was $\sim 0.05 \mathrm{~K}\left(T_{\mathrm{mb}}\right)$.

\subsection{OVRO Maps}

In Figures $1 a$ and $1 b$ we show the CS (2-1) integrated intensity mosaic overlaid on our $3 \mathrm{~mm}$ continuum map (TS98) and the CS (2-1) mean velocity mosaic, respectively. In Figure $1 a$ some of the brightest CS (2-1) features are labeled to simplify discussion; the location of the near-IR cluster surrounding SVS-2 and SVS-20 (Strom et al. 1976) is also indicated. In Figure $1 b$, the orientations of the jets emanating from SMM1, SMM3, SMM4, and A3 as well as the near-IR reflection nebula associated with SMM5 are marked.

At most, $20 \%$ of the flux detected in single-dish maps of the optically thick and extended CS (2-1) emission (see McMullin et al. 1994) is recovered. There is therefore little point in comparing the CS and the optically thin $3 \mathrm{~mm}$ emission (TS98). However, the molecular line observations provide information about the outflows emanating from the various millimeter sources; the abundance of CS can increase by a factor of almost 100 in outflows (Bachiller \& Pérez Gutiérrez 1997), and most of the clumps in Figure $1 a$ are likely to be compact enhancements in shocked regions. In most cases the CS line widths are relatively broad, $\geq 2-3 \mathrm{~km} \mathrm{~s}^{-1}$, and mean velocities differ by $3-4 \mathrm{~km} \mathrm{~s}^{-1}$ from the systemic velocity of the Serpens core (Fig. $1 b$ ). Strong and broad $\mathrm{CH}_{3} \mathrm{OH}$ emission, typical of shocked material, was also seen in our follow-up observations (Fig. 2) and coincides with the CS features. By contrast, $\mathrm{N}_{2} \mathrm{H}^{+}$ emission is spatially coincident with the continuum sources, as expected for a tracer of cores and envelopes. 

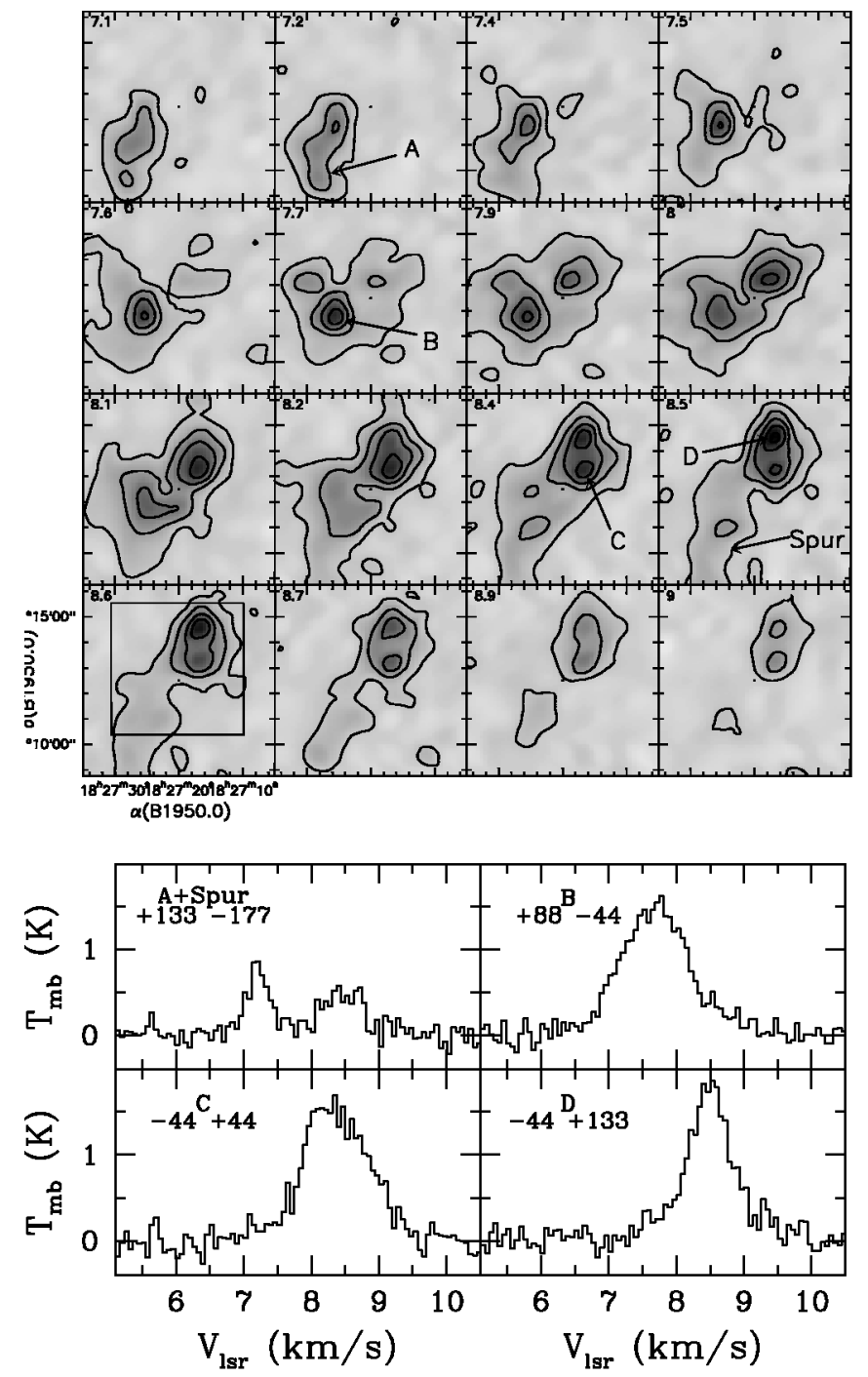

FIG. 3.-Top: FCRAO/SEQUOIA $\mathrm{N}_{2} \mathrm{H}^{+}\left(1_{01}-0_{12}\right)$ channel maps. The five main kinematical structures, A, B, C, D, and the "spur," are each labeled at their approximate $V_{\mathrm{LSR}}$, which is given in the top left corner of each panel. Contour levels are $0.18-2.18$ by $0.36 \mathrm{~K}$. The square in the bottom left channel shows the area mapped at OVRO. Bottom: $\mathrm{N}_{2} \mathrm{H}^{+}\left(1_{01}-0_{12}\right)$ spectra observed toward the four cores A, B, C, and D, as defined in Fig. 3. In each panel the offset in arcseconds from position $\alpha=18^{\mathrm{h}} 27^{\mathrm{m}} 20^{\mathrm{s}}, \delta=1^{\circ} 12^{\prime} 30^{\prime \prime}$ (B1950.0) is given.

\subsection{FCRAO $\mathrm{N}_{2} \mathrm{H}^{+}$Map}

Figure 3 shows the FCRAO/SEQUOIA large-scale channel maps of the $\mathrm{N}_{2} \mathrm{H}^{+}\left(1_{01}-0_{12}\right)$ isolated hyperfine component. Four cores, labeled $\mathrm{A}, \mathrm{B}, \mathrm{C}$, and $\mathrm{D}$ in order of increasing $V_{\mathrm{LSR}}$, and an extended, lower surface brightness "spur" can be identified. The spur stretches from the northwest $(\mathrm{C}, \mathrm{D})$ to southeast (A, $\mathrm{B})$ cores and continues beyond these. Cores A and B are spa- tially associated with the SMM3/SMM4 region, while $\mathrm{C}$ and $\mathrm{D}$ encompass the SMM1/S68N region. Spectra at the emission peaks in A, B, C, and D are also presented in Figure 3. These positions are given in Table 1 as offsets from $\alpha=$ $18^{\mathrm{h}} 27^{\mathrm{m}} 20^{\mathrm{s}}, \delta=1^{\circ} 12^{\prime} 30^{\prime \prime}$. Also listed are the $V_{\mathrm{LSR}}$, line width $\Delta V$, and virial mass $M_{\text {vir }}$ for each core. The fraction of the total $\mathrm{N}_{2} \mathrm{H}^{+}$flux observed with the array is $f_{\text {OvRo }} ; M_{d}$ is the total mass of $3 \mathrm{~mm}$ continuum cores within each gaseous core (TS98), and $M_{*}$ is the total mass of YSOs, derived assuming a mean stellar mass of $0.3 M_{\odot}$ (Giovannetti et al. 1998; Kaas 1999). The FCRAO and OVRO values of $\Delta V$ and $V_{\text {LSR }}$ are consistent, suggesting that the array is detecting a fraction of the emission from extended envelopes, rather than the compact cores found by TS98.

\subsection{Identification of Outflow Sources}

The powering sources of the CS outflows were identified from published optical, IR, and millimeter observations and are represented by diamonds in Figure 1. Features CS3 and $\mathrm{CS} 4$ as well as $\mathrm{CH}_{3} \mathrm{OH}$ emission (Fig. 2) suggest a compact outflow at P.A. $\sim 140^{\circ}$, centered on the $\mathrm{S} 68 \mathrm{~N}$ continuum source (see Wolf-Chase et al. 1998). CS2 could be either an extension of the S68N flow or the counterflow of the $\mathrm{H}_{2}$ jet from millimeter source A3 (Hodapp 1999; TS98). Based on its alignment with an $\mathrm{H}_{2}$ jet (Hodapp 1999) and the locations of HH 460 and a CO (2-1) bullet (Davis et al. 1999), we associate CS1 with an outflow from SMM1. CS6, CS7, and CS8 probably mark the interaction of the counterflow from SMM1 with the B molecular core. All these outflows are oriented along P.A. $\sim 140^{\circ}$. A double-peaked CS component reported by Williams \& Myers (1999) at the position of CS1 was not detected; it is probably extended and resolved out in our observations.

In the southeast, CS9 and $\mathrm{CS} 10$ are oriented like $\mathrm{CH}_{3} \mathrm{OH}$ (Fig. 2) and $\mathrm{H}_{2}$ knots near SMM4 (Eiroa et al. 1997) along P.A. $\sim 180^{\circ}$ and are probably part of an outflow centered on that source (see Hogerheijde et al. 1999). Likewise, CS12 is elongated along P.A. $\sim 170^{\circ}$ as are a chain of $\mathrm{H}_{2}$ knots associated with SMM3 (Herbst et al. 1997). Davis et al. (1999) noted that two Herbig-Haro objects and SMM3 are aligned along a position angle that is almost orthogonal to the chain of $\mathrm{H}_{2}$ knots. However, the kinematic properties of the knots suggest no physical connection with the Herbig-Haro objects.

\section{DISCUSSION}

Our new interferometer and single-dish maps of the Serpens core indicate subclustering at an early epoch of cluster formation. Three separate properties argue for subclustering, spatial segregation, outflow orientations, and circumcluster gas kinematics. Approximately one-third of the near-IR cluster members are concentrated in a $\sim 0.1$ pc radius region surrounding SVS-2 and SVS-20 (Giovannetti et al. 1998; Kaas 1999), while the millimeter and submillimeter sources are largely con-

TABLE 1

$\mathrm{N}_{2} \mathrm{H}^{+}(1-0)$ Cores Parameters

\begin{tabular}{ccccccccc}
\hline \hline Name & $\begin{array}{c}\Delta \alpha \\
(1950)\end{array}$ & $\begin{array}{c}\Delta \delta \\
(1950)\end{array}$ & $\begin{array}{c}V_{\mathrm{LSR}} \\
\left(\mathrm{km} \mathrm{s}^{-1}\right)\end{array}$ & $\begin{array}{c}\Delta V \\
\left(\mathrm{~km} \mathrm{~s}^{-1}\right)\end{array}$ & $\begin{array}{c}M_{\text {vir }} \\
\left(M_{\odot}\right)\end{array}$ & $f_{\text {ovRo }}$ & $\begin{array}{c}M_{d} \\
\left(M_{\odot}\right)\end{array}$ & $\begin{array}{c}M_{*} \\
\left(M_{\odot}\right)\end{array}$ \\
\hline $\mathrm{A} \ldots \ldots \ldots$ & 133 & -177 & 7.15 & 0.7 & $\ldots$ & $\ldots$ & $\ldots$ & $\ldots$ \\
$\mathrm{B} \ldots \ldots \ldots$ & 88 & -44 & 7.68 & 1.2 & 30 & $\sim 20 \%$ & 12 & 7 \\
$\mathrm{C} \ldots \ldots \ldots$ & -44 & 44 & 8.35 & 1.1 & 18 & $\ldots$ & 14 & 2 \\
$\mathrm{D} \ldots \ldots \ldots$ & -44 & 133 & 8.49 & 0.8 & 9 & $\leq 5 \%$ & 5.5 & 2 \\
Spur $\ldots \ldots$ & $\ldots$ & $\ldots$ & 8.32 & 0.7 & $\ldots$ & $\ldots$ & $\ldots$ & $\ldots$ \\
\hline
\end{tabular}


centrated in the southeast $(\mathrm{A}, \mathrm{B})$ and northwest $(\mathrm{C}, \mathrm{D})$ fragments (TS98; Davis et al. 1999). The orientations of the outflows observed in the southeast and northwest are quite different. In the northwest, all three flows are oriented along P.A. $\sim 140^{\circ}$, as is the near-IR reflection nebula centered on SMM5 (Kaas 1999; see also Fig. 1b). In the southeast, the two outflows are aligned approximately north-south, with mean P.A. $\sim 175^{\circ}$. In Figure 3, the northwest and southeast subclusters are embedded in discrete $\mathrm{N}_{2} \mathrm{H}^{+}(1-0)$ clumps, separated in velocity by $\sim 1 \mathrm{~km} \mathrm{~s}^{-1}$ (Table 1 ). Each clump comprises two cores: peak velocities of cores $\mathrm{A}$ and $\mathrm{B}$ differ by only 0.5 $\mathrm{km} \mathrm{s}^{-1}(\sim 1 / 2 \Delta V), \mathrm{C}$ and $\mathrm{D}$ by $0.1 \mathrm{~km} \mathrm{~s}^{-1}(\sim 1 / 10 \Delta V)$. Thus, the spatially distinct clumps are also kinematically separated, while there is reasonable internal velocity coherence.

It appears that the Serpens core encompasses at least three substructures - the northwest and southeast subclusters and the near-IR cluster. Star formation is currently occurring simultaneously in the northwest and southeast subclusters, both of which contain roughly equal fractions of prestellar, protostellar, and IR sources. The near-IR cluster is probably more evolved, but we see no evidence of the progressive pattern of star formation proposed by Casali et al. (1993). The kinematics and outflow orientations indicate that each subcluster originated in a separate fragment of the cloud which subsequently fragmented into the smaller cores seen in the $\mathrm{N}_{2} \mathrm{H}^{+}$(1-0) maps.
Within these are the $3 \mathrm{~mm}$ continuum cores that are likely progenitors of single stellar systems (TS98). Taken together, these observations are consistent with either the hierarchical fragmentation picture advocated by Elmegreen (1997, 1999) or the spontaneous fragmentation suggested by Myers (1998) for the formation of stellar clusters and provide the first observational support for hierarchical fragmentation within a cluster-forming core. We note that while the mean stellar/protostellar density of the entire Serpens core is $~ 400-800$ stars $\mathrm{pc}^{-3}$, most of the protocluster members are within the three subclusters where densities reach 2000-4000 stars $\mathrm{pc}^{-3}$. It is very likely that, within a few million years, the cluster will evolve to a size and mean density very similar to those of embedded clusters around intermediate-mass stars.

We thank Cathie Clarke and the referee, Paul Ho, for comments that much improved this Letter. The Owens Valley millimeter-wave array is supported by NSF grant AST 96-13717. Research on young star and disk systems is also supported by the Norris Planetary Origins Project and NASA's Origins of Solar Systems program (through grant NAGW-4030). The FCRAO observations were supported by NSF grant AST 9725951. J. S. O. thanks the Cornell University Department of Astronomy for continuing support and warm hospitality.

\section{REFERENCES}

Bachiller, R., \& Pérez Gutiérrez, M. 1997, ApJ, 487, L93

Bate, M. R., Clarke, C. J., \& McCaughrean, M. 1998, MNRAS, 297, 1163

Bonnell, I. A., Bate, M. R., Clarke, C. J., \& Pringle, J. E. 1997, MNRAS, 285, 201

Bonnell, I. A., Bate, M. R., \& Zinnecker, H. 1998, MNRAS, 298, 93

Casali, M. M., Eiroa, C., \& Duncan, W. D. 1993, A\&A, 275, 195

Clarke, C. J., Bonnell, I. A., \& Hillenbrand, L. A. 2000, in Protostars and Planets IV, ed. V. Mannings, A. Boss, \& S. S. Russell (Tucson: Univ. Arizona Press), 151

Davis, C. J., Matthews, H. E., Ray, T. P., Dent, W. R. F., \& Richer, J. S. 1999, MNRAS, 309, 141

de Lara, E., Chavarria-K. C., \& López-Molina, G. 1991, A\&A, 243, 139

Eiroa, C., \& Casali, M. M. 1992, A\&A, 262, 468

Eiroa, C., Palacios, J., Eislöffel, J., Casali, M. M., \& Curiel, S. 1997, in LowMass Star Formation from Infall to Outflow, ed. F. Malbet \& A. Castets (Grenoble: Obs. Grenoble), 103

Elmegreen, B. G. 1997, ApJ, 486, 944

. 1999, ApJ, 515, 323

Giovannetti, P., Caux, E., Nadeau, D., \& Monin, J.-L. 1998, A\&A, 330, 990

Herbst, T. M., Beckwith, S. V. W., \& Robberto, M. 1997, ApJ, 486, L59

Hodapp, K. W. 1999, AJ, 118, 1338

Hogerheijde, M. R., van Dishoeck, E. F., Salverda, J. M., \& Blake, G. A. 1999, ApJ, 513, 350

Hurt, R. L., \& Barsony, M. 1996, ApJ, 460, L45

Kaas, A. A. 1999 , AJ, 118,558
Kroupa, P., Petr, M. G., \& McCaughrean, M. J. 1999, NewA, 4, 495

McMullin, J. P., Mundy, L. G., Wilking, B. A., Hezel, T., \& Blake, G. A. 1994, ApJ, 424, 222

Meyer, M. R., Adams, F. C., Hillenbrand, L. A., Carpenter, J. M., \& Larson, R. B. 2000, in Protostars and Planets IV, ed. V. Mannings, A. P. Boss, \& S. S. Russell (Tucson: Univ. Arizona Press), 121

Motte, F., André, P., \& Neri, R. 1998, A\&A, 336, 150

Myers, P. C. 1998, ApJ, 496, L109

Palla, F., \& Stahler, S. 1999, ApJ, 525, 772

Rodríguez, L. F., Curiel, S., Moran, J. M., Mirabel, I. F., Roth, M., \& Garay, G. 1989, ApJ, 346, L85

Salpeter, E. E. 1955, ApJ, 121, 161

Scalo, J. M. 1986, Fundam. Cosmic Phys., 11, 1

Stahler, S. W., Palla, F., \& Ho, P. T. P. 2000, in Protostars and Planets IV, ed. V. Mannings, A. P. Boss, \& S. S. Russell (Tucson: Univ. Arizona Press), 327

Strom, S. E., Vrba, F. J., \& Strom, K. M. 1976, AJ, 81, 638

Testi, L., Palla, F., \& Natta, A. 1999, A\&A, 342, 515

Testi, L., \& Sargent, A. I. 1998, ApJ, 508, L91 (TS98)

. 2000, in Imaging at Radio through Submillimeter Wavelengths, ed. J. Mangum \& S. Radford (San Francisco: ASP), in press

White, G. J., Casali, M. M., \& Eiroa, C. 1995, A\&A, 298, 594

Williams, J. P., \& Myers, P. C. 1999, ApJ, 518, L37

Wolf-Chase, G. A., Barsony, M., Wootten, H. A., Ward-Thompson, D., Lowrance, P. J., Kastner, J. H., \& McMullin, J. P. 1998, ApJ, 501, L193 